\title{
Über einen Satz von Jacob Steiner
}

\author{
Adolf Schleiermacher \\ Adolf Schleiermacher hat in Frankfurt am Main Mathematik und Physik studiert. Im \\ Jahr 1968 hat er bei Ruth Moufang promoviert. Danach war er u.a. als Lecturer am \\ Imperial College in London tätig. Von 1975 bis 1999 arbeitete er in der Industrie. Er \\ fühlt sich dem Kreis von Mathematikern um Reinhold Baer verbunden.
}

\section{Einleitung}

Ein Hyperboloid ist eine Fläche zweiten Grades im dreidimensionalen projektiven Raum, die zwei Scharen von Geraden enthält, derart, daß jede Gerade der einen Schar zu allen übrigen Geraden dieser Schar windschief liegt, aber alle Geraden der andern Schar schneidet. Die Geraden der beiden Scharen nennt man auch Erzeugende des Hyperboloids. Hyperboloide gibt es nur bei kommutativem Koordinatenkörper (s. Herzer [8]). Im affinen Raum entstehen aus einem Hyperboloid zwei mögliche Typen von Flächen, je nachdem ob die unendlich ferne Ebene das Hyperboloid berührt oder schneidet: im ersten Fall das hyperbolische Paraboloid, im zweiten Fall das einschalige Hyperboloid.

Nach einem Satz von Jacob Steiner (s. [13] oder [14, S. 316]) sind bekanntlich die Höhen eines Tetraeders in einer der beiden Erzeugendenscharen eines einschaligen Hyperboloids enthalten, vorausgesetzt das Tetraeder erfüllt gewisse Bedingungen, die sicherstellen, daß von den Höhen keine zwei in einer Ebene liegen. Einen analytischen Beweis dieses Satzes hat Otto Hermes [7] gegeben.

Ein Satz von Steiner besagt, daß die vier Höhen eines Tetraeders in einem Hyperboloid liegen. Im nachfolgenden Beitrag werden die Bedingungen der Gültigkeit dieses Satzes in dem allgemeineren Kontext eines affinen Raumes mit Orthogonalität im Sinne von Lenz [10] untersucht. Als notwendige und hinreichende Bedingungen ergeben sich einige einfache geometrische Aussagen, wie z.B. der Höhensatz, die ihrerseits alle damit äquivalent sind, daß die Polarität in der unendlich fernen Ebene durch eine symmetrische Bilinearform induziert wird. Eine entsprechende Untersuchung wird für das projektive Analogon durchgeführt. Die diesbezüglichen Ergebnisse sind weitgehend analog zum affinen Fall. 
Es ist der Zweck dieser Note, den Steinerschen Satz in einen allgemeineren Zusammenhang zu stellen. Dazu betrachten wir einen affinen Raum mit einer Orthogonalität zwischen den Geraden im Sinne von Lenz [10]. Eine solche Orthogonalität mit den von Lenz geforderten natürlichen Eigenschaften läßt sich auf eine Polarität in der unendlich fernen Ebene zurückführen (s. Lenz [10]). Analysiert man den Satz von Steiner in diesem Zusammenhang, so wird man zwanglos auf eine Reihe einfacher geometrischer Aussagen geführt, die einerseits alle mit dem Satz selber und andererseits damit äquivalent sind, daß die Polarität in der unendlich fernen Ebene durch eine symmetrische Bilinearform gegeben ist. $\mathrm{Zu}$ ganz ähnlichen Ergebnissen gelangt man, wenn man den Satz in projektiven Räumen mit Polarität untersucht.

Die Ergebnisse sind zusammengefaßt in §6, Satz 1 für den affinen Fall und in §7, Satz 2 für den projektiven Fall. In den Paragraphen 2-5 stellen wir bekannte Resultate zusammen, die später benötigt werden. Die Beweise der Sätze 1 und 2 sind überwiegend synthetischer Natur und fußen auf den elementarsten Eigenschaften des Senkrechtstehens.

\section{Projektive Räume mit Polarität}

Sei $\mathcal{P}$ ein Desarguesscher projektiver Raum der endlichen Dimension $n \geq 2$ mit vorgegebener Polarität $\pi$. Die Polarität $\pi$ läßt sich durch eine nichtausgeartete Semibilinearform $f$ des zugrundeliegenden Vektorraums $V$ darstellen. Den zugehörigen Koordinatenschiefkörper bezeichnen wir mit $K$, den zugehörigen Antiautomorphismus von $K$ mit $\sigma$. Damit wirklich eine Polarität dargestellt wird, muß die Form $f$ noch die zusätzliche Eigenschaft $f(x, y)=0 \rightarrow f(y, x)=0$ haben (s. Baer [2]). Wenn $K$ kommutativ ist, kann $\sigma$ die Identität sein. Die Polaritäten, für die das zutrifft, lassen sich durch folgende geometrische Eigenschaft gemeinsam charakterisieren: sie bilden eine und damit jede Punktreihe $g$ projektiv auf das zugehörige Hyperebenenbüschel ab (s. Lenz [11]). Sie werden daher projektive Polaritäten genannt.

Wie üblich definiert man: Ein Punkt $P$ ist konjugiert zu einem Punkt $Q$, wenn $P$ auf der Polaren von $Q$ liegt. Eine Hyperebene $\alpha$ steht senkrecht auf einer Hyperebene $\beta$, wenn $\alpha$ den Pol von $\beta$ enthält. Wir schreiben hierfür $P \sim Q$ bzw. $\alpha \perp \beta$. Die Beziehungen $P \sim Q$ und $\alpha \perp \beta$ sind bekanntlich symmetrisch.

Sei $\alpha$ eine Hyperebene, die ihren Pol $P=\pi(\alpha)$ nicht enthält. Bilden wir jeden Unterraum $X$ von $\alpha$ auf $\pi(X) \cap \alpha$ ab, so erhalten wir eine Polarität von $\alpha$. Diese Polarität bezeichnen wir mit $\pi_{\alpha}$. Dual hierzu können wir die Gesamtheit der Unterräume betrachten, die den Punkt $P$ enthalten. Diese Unterräume bilden ebenfalls einen projektiven Raum der Dimension $n-1$. Wir nennen ihn den mit dem Büschel mit Träger $P$ assoziierten Raum und bezeichnen ihn mit $\mathcal{E}(P)$. Ist $P \in X$, so ist $\pi(X) \subseteq \alpha$. Die Abbildung $X \rightarrow \pi(X) \cup P$ ist eine Polarität des Büschelraums $\mathcal{E}(P)$. Diese Polarität bezeichnen wir mit $\pi_{(P)}$.

Die Abbildung $\varphi: X \rightarrow X \cup P$ ist ein Isomorphismus der Hyperebene $\alpha$ auf den Raum $\mathcal{E}(P)$, der mit den induzierten Polaritäten vertauschbar ist, für den also $\varphi \pi_{\alpha}(X)=$ $\pi_{(P)} \varphi(X)$ für alle Unterräume $X$ von $\alpha$ gilt.

\section{Affine Räume mit Orthogonalität}

Sei $\mathcal{A}$ ein dreidimensionaler affiner Raum mit einer Beziehung des Senkrechtstehens von Geraden im Sinne von Lenz [10]. Sind $g$ und $h$ Geraden, so wird ihr Senkrechtstehen 
durch $g \perp h$ ausgedrückt. Sind $g$ und $h$ parallel, so schreiben wir $g \| h$. Es wird nicht verlangt, daß aufeinander senkrecht stehende Geraden einen Punkt gemeinsam haben. Die Beziehung des Senkrechtstehens soll die folgenden Axiome erfüllen:
Ia) Aus $a \perp b$ folgt $b \perp a$.
Ib) Aus $a \perp b$ und $b \| c$ folgt $a \perp c$.
II) Ist $O A \perp O B, O A \perp O C, D \in B C, D \neq O$, so ist $O A \perp O D$.

Geraden, die auf sich selber senkrecht stehen, werden isotrop genannt.

IIIa) Ist die Gerade $A B$ nicht-isotrop, so liegt auf jeder von $A B$ verschiedenen Geraden durch $B$, die nicht auf $A B$ senkrecht steht, ein Punkt $C$ mit $A C \perp A B$.

IIIb) Ist die Gerade $A B$ isotrop, so liegt auf jeder $A B$ nicht schneidenden und nicht auf $A B$ senkrechten Geraden ein Punkt $C$ mit $A B \perp A C$.

IV) Keine Gerade steht auf allen Geraden senkrecht.

Wie Lenz gezeigt hat, läßt sich dann in der unendlich fernen Ebene eine Polarität $\pi$ einführen mit der Eigenschaft, daß zwei Geraden $g$ und $h$ genau dann aufeinander senkrecht stehen, wenn ihre unendlich fernen Punkte zueinander bezüglich der Polarität $\pi$ konjugiert sind.

Aus Axiom II) zusammen mit Ib) ergibt sich die weitere Aussage:

V) Steht eine Gerade auf zwei nichtparallelen Geraden einer Ebene senkrecht, so steht sie auf allen Geraden dieser Ebene senkrecht.

Definition. Eine Gerade $g$ steht auf einer Ebene $\alpha$ senkrecht, wenn sie auf allen Geraden der Ebene senkrecht steht. Wir schreiben hierfür $g \perp \alpha$.

Eine Ebene $\alpha$ steht auf einer Ebene $\beta$ senkrecht, wenn sie auf einer in $\beta$ enthaltenen Geraden senkrecht steht. Wir schreiben hierfür $\alpha \perp \beta$.

Aus der Tatsache, daß die Orthogonalität durch eine Polarität der unendlich fernen Ebene gegeben ist, folgen noch die Aussagen:

VI) Zu einer beliebigen Ebene $\alpha$ von $\mathcal{A}$ existiert durch einen beliebig gegebenen Punkt $P$ eine eindeutig bestimmte Gerade, die senkrecht auf $\alpha$ steht.

Wir nennen diese Gerade das Lot auf die Ebene $\alpha$ durch den Punkt $P$. Eine Ebene wird isotrop genannt, wenn sie eines ihrer Lote enthält.

VII) Für beliebige Ebenen $\alpha$ und $\beta$ gilt: Aus $\alpha \perp \beta$ folgt $\beta \perp \alpha$.

VIII) Wenn eine Ebene $\alpha$ auf zwei nicht-parallelen Ebenen $\beta$ und $\gamma$ senkrecht steht, so steht die Schnittgerade $g=\beta \cap \gamma$ auf $\alpha$ senkrecht.

\section{Der affine Satz vom Höhenschnittpunkt}

Sei $\mathcal{E}$ eine beliebige affine Ebene. Ist $g$ eine Gerade, so bezeichnen wir mit $\bar{g}$ ihren unendlich fernen Punkt. Es sei nun $\varphi$ eine involutorische Abbildung oder die Identität auf der Menge der unendlich fernen Punkte. Wir definieren eine Beziehung des Senkrechtstehens 
zwischen Geraden durch $g \perp_{\varphi} h$, wenn $\bar{h}=\varphi(\bar{g})$. Dann gibt es zu einer beliebigen Geraden $g$ durch einen beliebigen Punkt $P$ ein eindeutig bestimmtes Lot, d.h. eine Gerade $h$, so daß $g \perp_{\varphi} h$. Zum Höhensatz in dieser Situation vergleiche man die Untersuchungen von Baer [3].

Bemerkung. Ist $\varphi$ die identische Abbildung, so ist der Höhensatz, wie man leicht sieht, äquivalent mit der Aussage, daß in jedem Parallelogramm auch die Diagonalen parallel sind.

Hilfssatz. Wenn in $\mathcal{E}$ bezüglich der Relation $\perp_{\varphi}$ der Satz vom Höhenschnittpunkt gilt, so ist $\varphi$ eine Projektivität.

Beweis. Wir bezeichnen mit $[x X]$ die Perspektivität $Y \rightarrow Y X$ für $Y \in x$ und mit $[X x]$ deren Inverse. Wir benutzen eine Idee von Lenz [11, S. 65].

Seien $l$ die unendlich ferne Gerade der Ebene $\mathcal{E}, P$ ein fester Punkt und $a, b$ zwei nicht aufeinander senkrecht stehende Geraden durch den Punkt $P$. Wir setzen $A=\varphi(\bar{a})$ und $B=\varphi(\bar{b})$. Da $a$ und $b$ nicht aufeinander senkrecht stehen, ist $A \neq \bar{b}$ und $B \neq \bar{a}$. Wir wählen eine feste, nicht durch $P$ gehende Gerade $g$ durch den unendlich fernen Punkt $A$ und setzen $G=g \cap b$. Sei nun $h$ eine variable Gerade durch den Punkt $P$. Wir nehmen zunächst an, daß $h$ nicht durch $A$ und auch nicht durch $B$ geht. Dann läßt sich aufgrund des Höhensatzes das Bild $\varphi(\bar{h})$ des unendlich fernen Punktes $\bar{h}$ leicht konstruieren. Sei nämlich $S=h \cap g$ und $T=B S \cap a$. Im Dreieck $P G T$ ist $S$ der Schnittpunkt der Höhen durch den Punkt $G$ und durch den Punkt $T$. Also ist $h=P S$ die dritte durch $P$ gehende Höhe, und es folgt $h \perp G T$. Somit ist $\varphi(\bar{h})$ der unendlich ferne Punkt der Geraden $G T$. Es folgt aus der Konstruktion, daß $\varphi$ mit der Projektivität $\psi=[l P][P g][g B][B a][a G][G l]$ in allen Punkten $\bar{h}$ übereinstimmt. Da $\varphi$ offenbar auch mit $\psi$ in den Punkten $A$ und $B$ übereinstimmt, ist $\varphi$ eine Projektivität.

\section{Der projektive Satz vom Höhenschnittpunkt}

Sei $\mathcal{E}$ eine beliebige projektive Ebene mit fest gegebener Polarität $\pi$. Wie im Desarguesschen Fall läßt sich eine Beziehung des Senkrechtstehens zwischen Geraden definieren: die Gerade $g$ steht auf der Geraden $h$ senkrecht, wenn $h$ durch den Pol $\pi(g)$ von $g$ geht. Wir sagen dann auch: $g$ ist ein Lot auf $h$, und schreiben hierfür $g \perp h$. Die Relation $g \perp h$ ist symmetrisch.

In einem Dreieck heißt jedes Lot durch eine Ecke auf die gegenüberliegende Seite eine Höhe. Um die Höhen eindeutig zu machen, betrachtet man nur Dreiecke, in denen keine Ecke gleich dem Pol der gegenüberliegenden Seite ist. In Dreiecken mit zwei aufeinander senkrecht stehenden Seiten, ist die Ecke, wo diese aufeinandertreffen, trivialerweise ein Höhenschnittpunkt. Natürlich gilt der Satz vom Höhenschnittpunkt nicht in jeder projektiven Ebene mit Polarität. In einer Desarguesschen Ebene ist er, wie Lenz gezeigt hat (s. [11, S. 65]), damit gleichwertig, daß der Koordinatenkörper kommutativ ist und die Polarität durch eine symmetrische Bilinearform induziert wird. Ob der Satz von Desargues aus dem Höhensatz gefolgert werden kann, ist ein unseres Wissens noch offenes Problem.

Die folgende Aussage über vollständige Vierseite ist gleichwertig mit dem Satz vom Höhenschnittpunkt: 
Vierseitbedingung. Wenn in einem vollständigen Vierseit die Punkte in zwei gegenüberliegenden Paaren zueinander konjugiert sind, so sind sie es auch im dritten Paar.

Man erkennt die Äquivalenz mit dem Höhensatz am leichtesten durch Dualisieren: Stehen in einem vollständigen Viereck zwei Paare von Gegenseiten aufeinander senkrecht, so gilt dies auch für das dritte Paar. Diese Aussage ist, wie man sofort sieht, gleichwertig mit dem Höhensatz.

Unabhängig von der Polarität $\pi$ betrachten wir noch einen Sonderfall:

Hilfssatz. Sei $\mathcal{E}$ die Ebene über dem Körper mit zwei Elementen. Dann gilt für jede symmetrische Beziehung des Senkrechtstehens und in jedem Dreieck mit eindeutig bestimmten Höhen: die Höhen gehen durch einen gemeinsamen Punkt.

Beweis. Wir betrachten ein Dreieck $A B C$ und dürfen annehmen, daß keine zwei seiner Seiten aufeinander senkrecht stehen. Dann ist auch keine der Höhen gleich einer Dreiecksseite. Da nun aber die Ebene $\mathcal{E}$ nur sieben Punkte enthält, und auf jeder Geraden drei Punkte liegen, so gibt es nur einen einzigen Punkt $P$, der auf keiner Dreiecksseite liegt. Durch diesen Punkt müssen alle drei Höhen gehen.

\section{Der Satz von Steiner im affinen Raum}

In diesem Abschnitt betrachten wir einen dreidimensionalen affinen $\operatorname{Raum} \mathcal{A}$ mit einer Beziehung des Senkrechtstehens zwischen den Geraden, welche die Lenzschen Axiome erfüllt (s. §3).

Sei $P_{0} P_{1} P_{2} P_{3}$ ein Tetraeder. Durch jede Ecke $P_{i}$ geht ein eindeutig bestimmtes Lot auf die gegenüberliegende Seitenfläche $P_{j} P_{k} P_{l}$, wo $\{i, j, k, l\}=\{0,1,2,3\}$. Diese Lote nennt man Höhen. Wir bezeichnen die durch $P_{i}$ gehende Höhe mit $h_{i}$. Die Höhe $h_{i}$ schneidet nicht immer die gegenüberliegende Seitenfläche $P_{j} P_{k} P_{l}$, nämlich genau dann nicht, wenn die Seitenfläche $P_{j} P_{k} P_{l}$ isotrop ist. Wir nennen ein Tetraeder zulässig, wenn keine Kante auf der ihr gegenüberliegenden senkrecht steht.

Sei $P$; $a b c$ das Dreikant mit Spitze $P$ und Kanten $a, b$ und $c$. Ein Dreikant nennen wir zulässig, wenn keine seiner Kanten auf der ihr gegenüberliegenden Seitenfläche senkrecht steht.

6.1 Hilfssatz. Sei $P_{0}$; abc ein zulässiges Dreikant. Wenn es auf jeder Geraden mindestens vier Punkte gibt, so lassen sich Punkte $P_{1} \in a, P_{2} \in b$ und $P_{3} \in c$ finden, die zusammen mit $P_{0}$ ein zulässiges Tetraeder bilden.

Beweis. Wir wählen auf $a$ einen beliebigen von $P_{0}$ verschiedenen Punkt $P_{1}$. Sind dann $P_{2}$, $P_{2}^{\prime}$ von $P_{0}$ verschiedene Punkte auf $b$, so kann höchstens eine der Geraden $P_{1} P_{2}$ oder $P_{1} P_{2}^{\prime}$ senkrecht auf $c$ stehen. Denn andernfalls wäre $c \perp a \cup b$ entgegen unseren Annahmen. Also können wir einen Punkt $P_{2} \neq P_{0}$ auf $b$ wählen, so daß $P_{1} P_{2}$ nicht auf $c$ senkrecht steht. Auf der Geraden $c$ gibt es nun mindestens drei von $P_{0}$ verschiedene Punkte $P_{3}$, $P_{3}^{\prime}, P_{3}^{\prime \prime}$. Von den Geraden $P_{1} P_{3}, P_{1} P_{3}^{\prime}$ usw. kann wieder höchstens eine senkrecht auf $b$ stehen, und von den Geraden $P_{2} P_{3}, P_{2} P_{3}^{\prime}$ usw. kann auch nicht mehr als eine senkrecht auf 
$a$ stehen. Also können wir einen Punkt $P_{3}$ auf $c$ so wählen, daß $P_{1} P_{3}$ nicht senkrecht auf $b$ und $P_{2} P_{3}$ auch nicht senkrecht auf $a$ steht. Dann ist $P_{0} P_{1} P_{2} P_{3}$ ein zulässiges Tetraeder.

Der obige Hilfssatz ist im affinen Raum über dem Körper mit zwei oder drei Elementen nicht anwendbar. Betrachten wir zunächst den affinen Raum über dem Körper mit drei Elementen. Ist $f$ eine nichtausgeartete symmetrische Bilinearform des zugrundeliegenden Vektorraums vom Rang 3, so hat bezüglich einer geeigneten Basis $v_{1}, v_{2}, v_{3}$ die Form $f$ die Diagonalgestalt

$$
f(x, y)=x_{1} y_{1}+x_{2} y_{2}+x_{3} y_{3},
$$

wobei $x=\sum x_{i} v_{i}, y=\sum y_{i} v_{i}$ (s. Dickson [6, S. 158]). Man verifiziert nun leicht, daß z.B. die vier Punkte mit den Koordinatenvektoren $(0,0,0),(1,0,0),(0,-1,0)$ und $(1,1,1)$ ein zulässiges Tetraeder bilden. Es ist auch nicht schwierig, sich durch ein Beispiel zu überzeugen, daß Hilfssatz 6.1 in diesem Raum nicht gültig bleibt. Im affinen Raum über dem Körper mit zwei Elementen gibt es keine zulässigen Tetraeder (s. Bemerkung am Schluß von §7).

6.2 Hilfssatz. Die Höhen eines beliebigen Tetraeders haben unterschiedliche Fernpunkte, von denen keine drei kollinear liegen. Daher sind die Höhen untereinander verschieden, und keine drei von ihnen liegen parallel zur selben Ebene.

Beweis. Die Ferngeraden der vier Seitenflächen des Tetraeders befinden sich in allgemeiner Lage. Von den Polen dieser Ferngeraden liegen also keine drei kollinear. Die Pole entsprechen aber den Fernpunkten der Höhen.

6.3 Hilfssatz. Sei $i \neq j$ ein Indexpaar mit $0 \leq i, j \leq 3$. Genau dann liegen die Höhen $h_{i}$ und $h_{j}$ in einer Ebene, wenn die einander gegenüberliegenden Kanten $P_{i} P_{j}$ und $P_{k} P_{l}$ mit $i, j \neq k, l$ aufeinander senkrecht stehen.

Beweis. Es gelte $P_{k} P_{l} \perp P_{i} P_{j}$. Ist $h_{j}=P_{i} P_{j}$, so haben $h_{i}$ und $h_{j}$ den Punkt $P_{i}$ gemeinsam. Sei also $h_{j} \neq P_{i} P_{j}$, und sei $\beta$ die Ebene $P_{i} \cup h_{j}$. Nun gilt $P_{k} P_{l} \perp P_{i} P_{j}$ und $P_{k} P_{l} \perp h_{j}$, also folgt $P_{k} P_{l} \perp \beta$ nach $\S 3$. Dann steht die Ebene $\beta$ senkrecht auf jeder Ebene, die $P_{k} P_{l}$ enthält, mithin auf $P_{j} P_{k} P_{l}$. Daraus folgt, daß $\beta$ auch die Höhe $h_{i}$ enthält. Liege nun umgekehrt die Höhe $h_{i}$ mit $h_{j}$ in einer Ebene $\beta$. Die Ebene $\beta$ enthält die Gerade $P_{i} P_{j}$. Die Gerade $P_{k} P_{l}$ steht nun senkrecht auf den Höhen $h_{j}$ und $h_{i}$, die einander nicht parallel sein können. Also steht $P_{k} P_{l}$ senkrecht auf allen Geraden der Ebene $\beta$, mithin auch auf $P_{i} P_{j}$.

Tetraeder, in denen alle Paare gegenüberliegender Kanten aufeinander senkrecht stehen, heißen orthogonal.

6.4 Hilfssatz. In einem orthogonalen Tetraeder und nur in einem solchen gehen alle vier Höhen durch einen Punkt. 
Beweis. In einem orthogonalen Tetraeder schneiden sich nach den Hilfssätzen 6.2 und 6.3 die Höhen paarweise. Daraus folgt, daß sie alle vier durch einen Punkt gehen oder in einer Ebene liegen. Da letzteres nicht möglich ist, gehen sie durch einen Punkt.

Die Umkehrung folgt aus Hilfssatz 6.3.

Der Satz von Steiner läßt sich nun wie folgt aussprechen:

6.5 Satz von Steiner. In jedem zulässigen Tetraeder liegen die Höhen in einer von den zwei Erzeugendenscharen eines einschaligen Hyperboloids.

Der Satz von Steiner gilt natürlich nicht in jedem affinen Raum mit Orthogonalität, da die Existenz eines Hyperboloids schon den Satz von Pappos nach sich zieht. Das Hyperboloid kann übrigens, wenn es existiert, nur ein einschaliges Hyperboloid sein. Denn lägen die Höhen als Erzeugende in einem hyperbolischen Paraboloid, so enthielte dieses eine Gerade $g$ aus der zweiten Erzeugendenschar in der unendlich fernen Ebene. Die Höhen lägen dann alle parallel zu jeder Ebene mit Ferngerade $g$, was nach Hilfssatz 6.2 ausgeschlossen ist.

Nennen wir zwei in derselben Ebene liegende Geraden Treffgeraden (sie können sich schneiden oder parallel sein), so folgt aus dem Satz von Steiner insbesondere die folgende Aussage:

6.6 Steinersche Bedingung. Durch jede Ecke eines beliebigen zulässigen Tetraeders geht eine Treffgerade der vier Höhen.

Steiner hat gezeigt, daß diese Bedingung im euklidischen Raum erfüllt ist, und daraus auf die Existenz des Höhenhyperboloids geschlossen. Daneben interessieren uns noch die folgenden Bedingungen:

6.7 Affine Dreikantbedingung. In jedem zulässigen Dreikant haben die durch die Kanten gehenden und auf den gegenüberliegenden Seitenflächen senkrecht stehenden Ebenen eine Gerade gemeinsam.

6.8 Affine Tetraederbedingung. Wenn in einem Tetraeder zwei Paare von gegenüberliegenden Kanten aufeinander senkrecht stehen, so tun dies auch die Kanten des dritten Paares.

Vgl. hierzu den Satz in [12, S. 84], ebenso [9, S. 107]. Man erkennt sofort die enge Beziehung dieser Bedingung zum affinen Satz vom Höhenschnittpunkt. Man kann beide Aussagen in der folgenden Form zusammenfassen: Sind $A, B, C, D$ vier untereinander verschiedene Punkte und gilt $A D \perp B C$, sowie $B D \perp A C$, so folgt $C D \perp A B$. Auch lassen sich manche Beweise des Höhensatzes fast wörtlich auf die Tetraederbedingung übertragen (vgl. Berger [5, Bd I, 10.13.1]).

6.9 Joachimsthalsche Bedingung. Sei h eine der Höhen in einem zulässigen Tetraeder. Dann gibt es eine Treffgerade aller vier Höhen, die zu h parallel ist. 
Diese Bedingung liefert wegen Hilfssatz 6.2 immer vier verschiedene Treffgeraden.

Satz 1 Sei $\mathcal{A}$ ein dreidimensionaler affiner Raum mit Orthogonalität, $\pi$ die zugehörige Polarität der unendlich fernen Ebene $\mathcal{E}$ und $f$ eine in dem $\mathcal{A}$ zugrundeliegenden Vektorraum definierte Semibilinearform, welche $\pi$ induziert. Dann sind folgende Aussagen äquivalent:

i) In $\mathcal{A}$ ist die Steinersche Bedingung erfüllt.

ii) In $\mathcal{A}$ ist die affine Dreikantbedingung erfüllt.

iii) In der unendlich fernen Ebene $\mathcal{E}$ gilt bezüglich $\pi$ der projektive Satz vom Höhenschnittpunkt.

iv) In der unendlich fernen Ebene $\mathcal{E}$ ist die Vierseitbedingung erfüllt.

v) In $\mathcal{A}$ ist die affine Tetraederbedingung erfüllt.

vi) In jeder nicht-isotropen Ebene von $\mathcal{A}$ gilt bezüglich $f$ der affine Satz vom Höhenschnittpunkt.

vii) In $\mathcal{A}$ gilt der Satz von Pappos, und $f$ ist eine symmetrische Bilinearform.

viii) In $\mathcal{A}$ ist die Joachimsthalsche Bedingung erfüllt.

ix) In $\mathcal{A}$ gilt der Satz von Steiner.

Bevor wir zum eigentlichen Beweis kommen, benötigen wir noch einen Hilfssatz.

6.10 Hilfssatz. Im Dreieck $P_{0} P_{1} P_{2}$ existiere ein Höhenschnittpunkt $H$. Wenn dann die Höhe $h_{3}$ im Tetraeder $\Delta=P_{0} P_{1} P_{2} P_{3}$ durch den Punkt $H$ geht, so ist $\Delta$ ein orthogonales Tetraeder.

Beweis. Wir zeigen, daß zum Beispiel $P_{0} P_{3} \perp P_{1} P_{2}$ gilt. Die restlichen Orthogonalitätsbeziehungen folgen aus Symmetriegründen. Ist zunächst $h_{3}=P_{0} P_{3}$, so gilt die geforderte Beziehung trivialerweise. Also dürfen wir $h_{3} \neq P_{0} P_{3}$ annehmen. Dann bestimmen die Punkte $P_{0}, P_{3}, H$ eine Ebene und die Punkte $P_{0}, H$ eine Gerade. Es gilt nach Voraussetzung $P_{0} H \perp P_{1} P_{2}$ und $P_{3} H \perp P_{1} P_{2}$. Also steht die Gerade $P_{1} P_{2}$ auf zwei nichtparallelen Geraden der Ebene $P_{0} P_{3} H$ senkrecht. Mithin steht sie auf allen Geraden dieser Ebene senkrecht, also auch auf $P_{0} P_{3}$.

Beweis von Satz 1. i) $\rightarrow$ ii). Wir betrachten ein zulässiges Dreikant $P_{0}$; $a b c$. Wenn es Punkte $P_{1} \in a, P_{2} \in b$ und $P_{3} \in c$ gibt, die mit der Spitze $P_{0}$ zusammen ein zulässiges Tetraeder bilden, so existiert eine durch $P_{0}$ gehende Treffgerade der vier Höhen dieses Tetraeders. Sie sei mit $t$ bezeichnet. Ist $t$ von $a=P_{0} P_{1}$ verschieden, so enthält die Ebene $a \cup t$ auch die Höhe $h_{1}$, ist also identisch mit der Ebene $\alpha$ durch $a$, die auf $b \cup c=P_{0} P_{2} P_{3}$ senkrecht steht. Ist aber $t=a$, so enthält die Ebene $\alpha$ jedenfalls $t$. In ähnlicher Weise zeigen wir, daß die Ebene $\beta$ durch $b$, die auf $c \cup a$ senkrecht steht, und die Ebene $\gamma$ durch $c$, die auf $a \cup b$ senkrecht steht, die Treffgerade $t$ enthalten. Es gilt also die Aussage der Dreikantbedingung. 
Wenn es auf jeder Geraden von $\mathcal{A}$ mindestens vier Punkte gibt, folgt die Existenz der oben geforderten Punkte $P_{1}, P_{2}$ und $P_{3}$ aus Hilfssatz 6.1.

Wenn es auf jeder Geraden höchstens drei Punkte gibt, ist die obige Schlußweise nicht durchführbar. Dann ist aber $\mathcal{A}$ ein affiner Raum über dem Körper von zwei oder von drei Elementen. Der Koordinatenkörper von $\mathcal{A}$ ist dann jedenfalls kommutativ und die Form $f$ symmetrisch. Nach Lenz [11, Satz 19, S. 65] gilt in jeder Büschelebene dann der Höhensatz und dies ist nichts anderes als die affine Dreikantbedingung.

ii) $\rightarrow$ iii). Sei $A B C$ ein Dreieck in der unendlich fernen Ebene, in welchem keine Ecke mit dem Pol der Gegenseite zusammenfalle. Wir wählen in $\mathcal{A}$ einen beliebigen Punkt $P_{0}$. Dann bilden die Geraden $a=P_{0} A, b=P_{0} B$ und $c=P_{0} C$ ein zulässiges Dreikant. Den drei Ebenen durch die Kanten des Dreikants, die auf den gegenüberliegenden Seitenflächen senkrecht stehen, entsprechen als unendlich ferne Geraden die Höhen des Dreiecks $A B C$. Da die drei Ebenen nach Voraussetzung eine Gerade gemeinsam haben, gehen die Höhen durch den unendlich fernen Punkt dieser Geraden.

iii) $\rightarrow$ iv). Siehe $\$ 5$.

iv) $\rightarrow$ v). Die Ferngeraden der Seitenflächen eines Tetraeders bilden zusammen mit den Fernpunkten der Kanten in der unendlich fernen Ebene ein vollständiges Vierseit. Da nun Geraden genau dann aufeinander senkrecht stehen, wenn ihre Fernpunkte konjugiert sind, ergibt sich aus der Vierseitbedingung die affine Tetraederbedingung.

v) $\rightarrow$ vi). Sei $\alpha$ eine nicht-isotrope Ebene und $A B C$ ein Dreieck in $\alpha$. Wir können annehmen, daß das Dreieck nicht rechtwinklig ist. Sei $H$ der Schnittpunkt der beiden durch $A$ bzw. durch $B$ gehenden Höhen. Dann ist also $H$ von $A, B$ und $C$ verschieden. Wir errichten im Punkte $H$ das Lot auf die Ebene $\alpha$ und bezeichnen es mit $h$. Da $\alpha$ nicht-isotrop ist, liegt $h$ nicht in $\alpha$, und wir können auf $h$ einen Punkt $D$ außerhalb von $\alpha$ wählen.

Nun gilt $B C \perp h$ und $B C \perp A H$. Also folgt $B C \perp A \cup h$ und daher $B C \perp A D$. Weiterhin $A C \perp h$ und $A C \perp B H$, also $A C \perp B \cup h$ und daher $A C \perp B D$. Nach Voraussetzung gilt also auch $A B \perp C D$. Da auch $A B \perp h$, folgt also $A B \perp C \cup h$ und somit $A B \perp C H$. Daher ist $C H$ die dritte Höhe im Dreieck $A B C$ und vi) ist bewiesen.

vi) $\rightarrow$ vii). Da die Polarität der unendlich fernen Ebene kein Nullsystem sein kann, gibt es nicht-isotrope Ebenen. Sei $\alpha$ eine solche und $\bar{\alpha}$ die unendlich ferne Gerade von $\alpha$. Sei $\bar{x}$ ein beliebiger Punkt auf $\bar{\alpha}, \bar{y}$ der eindeutig bestimmte zu $\bar{x}$ bezüglich $\pi$ konjugierte Punkt auf $\bar{\alpha}$, d.h. $\bar{y}=\pi(\bar{x}) \cap \bar{\alpha}$. Dann ist die Abbildung $\bar{x} \rightarrow \bar{y}$ nach dem Hilfssatz in $\$ 4$ eine Projektivität, und daraus folgt, daß die Polarität $\pi$ die Punktreihe $\bar{\alpha}$ projektiv auf das Geradenbüschel mit Träger $\pi(\bar{\alpha})$ abbildet. Damit bildet sie jede Punktreihe auf das entsprechende Geradenbüschel projektiv ab, und weil $\pi$ kein Nullsystem sein kann, gilt vii).

vii) $\rightarrow$ iii). Dies gilt nach Lenz [11, Satz 19, S. 65].

Da nun die Aussagen iii)-vii) zyklisch auseinander folgen, sind sie untereinander äquivalent.

v) und vi) $\rightarrow$ viii). Es sei $P_{0} P_{1} P_{2} P_{3}$ ein zulässiges Tetraeder. Für eine beliebige Höhe in diesem Tetraeder ist zu beweisen, daß sie eine Parallele hat, die auch die übrigen Höhen 
trifft. Wir führen den Beweis nur für die Höhe $h_{3}$ durch. Für die übrigen Höhen geht er analog.

Betrachten wir zunächst den Fall, daß die Ebene $P_{0} P_{1} P_{2}$ nicht-isotrop ist. Es sei $H$ der Höhenschnittpunkt im Dreieck $P_{0} P_{1} P_{2}$ und $F_{3}$ der Fußpunkt der Höhe $h_{3}$. Beide sind eigentliche Punkte, die wegen Hilfssatz 6.10 nicht zusammenfallen. Die Parallele $l$ zu $h_{3}$ durch den Punkt $H$ ist jedenfalls eine Treffgerade zu $h_{3}$. Sei $p_{0}$ die durch den Punkt $P_{0}$ gehende Höhe im Dreieck $P_{0} P_{1} P_{2}$. Die Geraden $p_{0}$ und $P_{1} P_{2}$ stehen aufeinander senkrecht, ebenso die Geraden $l$ und $P_{1} P_{2}$. Daher steht $P_{1} P_{2}$ auf der Ebene $p_{0} \cup l$ senkrecht. Daraus folgt, daß die Ebenen $P_{1} P_{2} P_{3}$ und $p_{0} \cup l$ aufeinander senkrecht stehen, und mithin enthält die Ebene $p_{0} \cup l$ durch jeden in ihr liegenden Punkt ein Lot auf die Ebene $P_{1} P_{2} P_{3}$ (vgl. $\S 3$, VII). Das durch den Punkt $P_{0}$ gehende Lot ist die Höhe $h_{0}$, die also in $p_{0} \cup l$ enthalten ist. $h_{0}$ und $l$ sind Treffgeraden. Auf analoge Weise zeigt man, daß auch $h_{1}$ bzw. $h_{2}$ und $l$ Treffgeraden sind.

Nehmen wir nun an, die Ebene $P_{0} P_{1} P_{2}$ sei isotrop. Die Höhe $h_{3}$ ist in diesem Fall parallel zur Ebene $P_{0} P_{1} P_{2}$. Wir bezeichnen mit $q_{i}$ die Parallele zu $h_{3}$ durch den Punkt $P_{i}, i=$ $0,1,2$. Von den drei Geraden $q_{i}$ können höchstens zwei zusammenfallen. Ist zum Beispiel $q_{0}=q_{1}=P_{0} P_{1}$, so ist jede Ebene senkrecht zu $P_{0} P_{1}$ parallel zu $P_{0} P_{1} P_{2}$. Die Höhe $h_{2}$ liegt in der eindeutig bestimmten Ebene senkrecht zu $P_{0} P_{1}$, die durch $P_{2}$ geht. Dies ist aber die Ebene $P_{0} P_{1} P_{2}$. Also liegt $h_{2}$ in der Ebene $P_{0} P_{1} P_{2}$. Dann ist $q_{0}=q_{1}=$ $P_{0} P_{1}$ die gesuchte Treffgerade der vier Höhen. Wir können also die Geraden $q_{0}, q_{1}, q_{2}$ als untereinander verschieden annehmen.

Wir bezeichnen mit $\alpha_{i}$ die Ebene durch $P_{i}$, die auf $P_{k} P_{l}$ senkrecht steht, $i, k, l=0,1,2$ und $k, l \neq i$. Die Ebenen $\alpha_{i}, i=0,1,2$, sind untereinander und von $P_{0} P_{1} P_{2}$ verschieden und nicht parallel. Die Ebene $\alpha_{i}$ enthält die Gerade $q_{i}$. Die Ebenen $\alpha_{0}$ und $\alpha_{1}$ schneiden sich in einer Geraden $q$ parallel zu $h_{3}$, welche die Seitenfläche $P_{1} P_{2} P_{3}$ in einem Punkte $Q$ trifft. Wir wollen zeigen, daß auch die dritte Ebene $\alpha_{2}$ die Gerade $q$ enthält.

Es gilt $P_{0} Q \perp P_{1} P_{2}$ und $P_{1} Q \perp P_{0} P_{2}$. Also sind in dem Tetraeder $P_{0} P_{1} P_{2} Q$ auch die Kanten des dritten Paares orthogonal: $P_{2} Q \perp P_{0} P_{1}$. Daraus folgt, daß die Gerade $P_{0} P_{1}$ auf der Ebene $q_{2} \cup Q$ senkrecht steht, und somit $q_{2} \cup Q=\alpha_{2}$. Damit ist gezeigt, daß alle drei Ebenen $\alpha_{i}$ die Gerade $q$ enthalten. Die Ebene $\alpha_{i}$ enthält aber die Höhe $h_{i}, i=0,1,2$. Daher ist $q$ Treffgerade der drei Höhen $h_{0}, h_{1}, h_{2}$. Die Punkte $Q$ und $P_{3}$ können nicht zusammenfallen, weil das Tetraeder $P_{0} P_{1} P_{2} Q$ orthogonal, das Tetraeder $P_{0} P_{1} P_{2} P_{3}$ aber zulässig ist. Daher sind auch $q$ und $h_{3}$ Treffgeraden.

Um viii) $\rightarrow$ ix) zu beweisen, zeigen wir zunächst viii) $\rightarrow$ vi). Sei also $P_{0} P_{1} P_{2}$ ein Dreieck in einer nicht-isotropen Ebene $\alpha$. Wir benötigen einen Punkt $P_{3}$ außerhalb von $\alpha$, so daß die Punkte $P_{0}, P_{1}, P_{2}, P_{3}$ ein zulässiges Tetraeder bilden. Die Punkte $P_{3}$, für die eine der Relationen $P_{i} P_{3} \perp P_{j} P_{k}, i=0,1,2, j, k \neq i, 3$, besteht, liegen in drei Ebenen $\alpha_{i}$; dabei geht $\alpha_{i}$ durch den Punkt $P_{i}$ und steht senkrecht auf der Kante $P_{j} P_{k}$. Liegt also $P_{3}$ außerhalb von $\alpha, \alpha_{0}, \alpha_{1}$ und $\alpha_{2}$, so ist $P_{0} P_{1} P_{2} P_{3}$ ein zulässiges Tetraeder. Gibt es aber keinen Punkt außerhalb dieser vier Ebenen, so ist $\mathcal{A}$ der affine Raum über dem Körper mit zwei Elementen. In diesem Fall folgt der affine Satz vom Höhenschnittpunkt aus dem Hilfssatz in §5. Denn wir können die Beziehung des Senkrechtstehens von der affinen Ebene auf die projektive Ebene erweitern, indem wir jede Gerade als orthogonal zur uneigentlichen 
Geraden auffassen. Wir dürfen also annehmen, daß ein Punkt $P_{3}$ mit der gewünschten Eigenschaft existiert.

Im Tetraeder $P_{0} P_{1} P_{2} P_{3}$ existiert zur Höhe $h_{3}$ eine Parallele $l$, die alle vier Höhen trifft. Da $\alpha$ nicht-isotrop ist, so sind $h_{3}$ und $l$ nicht parallel zur Ebene $\alpha$. Somit existiert der Schnittpunkt $H=l \cap \alpha$. Sei $q_{0}$ die durch den Punkt $P_{0}$ gehende Höhe im Dreieck $P_{0} P_{1} P_{2}$. Wir wollen zeigen, daß $H \in q_{0}$.

Ist $H=P_{0}$, so ist das klar. Andernfalls existiert die Verbindungsgerade $p_{0}=P_{0} H$. Die Ebene $p_{0} \cup l$ steht senkrecht auf $\alpha=P_{0} P_{1} P_{2}$, da sie die Gerade $l$ enthält. Die Ebene $p_{0} \cup l$ enthält aber auch die Höhe $h_{0}$ des Tetraeders, denn $h_{0}$ und $l$ liegen als Treffgeraden in einer Ebene und $h_{0}$ enthält den Punkt $P_{0}$ der Ebene $p_{0} \cup l$. Somit steht die Ebene $p_{0} \cup l$ auch auf der Seitenfläche $P_{1} P_{2} P_{3}$ senkrecht. Daher steht die Schnittgerade $P_{1} P_{2}$ der beiden Ebenen $P_{0} P_{1} P_{2}$ und $P_{1} P_{2} P_{3}$ senkrecht auf der Ebene $p_{0} \cup l$. Insbesondere gilt $P_{1} P_{2} \perp p_{0}$, woraus $p_{0}=q_{0}$ folgt. Die Höhe $q_{0}$ geht also durch den Punkt $H$. Auf analoge Weise zeigt man, daß auch die beiden anderen Höhen im Dreieck $P_{0} P_{1} P_{2}$ durch den Punkt $H$ gehen. Damit gilt in jeder nicht-isotropen Ebene der affine Satz vom Höhenschnittpunkt, es gilt also vi). Wie wir bereits wissen, folgt aus vi) der Satz von Pappos. In einem Papposschen affinen Raum liegen vier paarweise windschiefe Geraden in einem Hyperboloid, wenn sie mindestens drei verschiedene Treffgeraden haben. Die Joachimsthalsche Bedingung liefert uns aber sogar in einem beliebigen zulässigen Tetraeder vier verschiedene Treffgeraden der vier Höhen $h_{i}$. Also liegen die Höhen als Erzeugende ein- und derselben Schar in einem Hyperboloid, und es gilt ix).

ix) $\rightarrow$ i) folgt aus der Definition eines Hyperboloids.

Damit ist Satz 1 bewiesen.

\section{Der Satz von Steiner im projektiven Raum}

In diesem Abschnitt betrachten wir einen dreidimensionalen projektiven Raum $\mathcal{P}$ mit vorgegebener Polarität $\pi$. Mit $V$ bezeichnen wir den zugrundeliegenden Vektorraum vom Rang vier und mit $f$ eine Semibilinearform, die $\pi$ induziert. Vermerkt sei noch, daß in dem projektiven Raum $\mathcal{P}$ von ungerader Dimension auch Nullsysteme möglich sind.

7.1 Definition. Wir nennen eine Gerade $g$ konjugiert zu einer Geraden $h$, wenn $h$ und $\pi(g)$ in einer Ebene liegen. Wir schreiben hierfür $g \sim h$.

Zur Terminologie vgl. [4, Vol. III, S. 34]). Es wird nicht verlangt, daß die Ebene eindeutig bestimmt ist. Es ist also auch der Fall $h=\pi(g)$ mit eingeschlossen. Die Beziehung $g \sim h$ ist offensichtlich symmetrisch.

7.2 Definition. Eine Gerade $g$ steht senkrecht auf einer Ebene $\alpha$, wenn sie durch den Pol der Ebene $\alpha$ geht. Wir schreiben hierfür $g \perp \alpha$.

Da $\pi(\alpha) \in g$ gleichwertig ist mit $\pi(g) \subseteq \alpha$, folgt aus obiger Definition: Wenn eine Gerade $g$ auf einer Ebene $\alpha$ senkrecht steht, so ist sie konjugiert zu allen Geraden der Ebene $\alpha$. Von dieser Aussage gilt auch die Umkehrung und zwar in der schärferen Form: 
7.3 Hilfssatz. Wenn eine Gerade zu drei nicht durch einen Punkt gehenden Geraden einer Ebene konjugiert ist, so steht sie auf der Ebene senkrecht.

Beweis. Seien $a, b, c$ drei Geraden einer Ebene $\alpha$, die nicht durch einen Punkt gehen, und sei $g$ eine zu $a, b$ und $c$ konjugierte Gerade. Die Gerade $\pi(g)$ liegt mit jeder der drei Geraden $a, b, c$ in einer gemeinsamen Ebene, hat also mit jeder von ihnen einen Punkt gemeinsam. Daraus folgt $\pi(g) \subseteq \alpha$ und $\pi(\alpha) \in g$. Somit ist $g \perp \alpha$.

7.4 Hilfssatz. Sei $\alpha$ eine Ebene und a eine auf $\alpha$ nicht senkrecht stehende Gerade. Dann sind die in $\alpha$ enthaltenen Geraden, die zu a konjugiert sind, genau die durch den Punkt $\pi(a \cup \pi(\alpha))=\pi(a) \cap \alpha$ gehenden.

Beweis. Da $a$ auf $\alpha$ nicht senkrecht steht, liegt der Punkt $\pi(\alpha)$ nicht auf $a$, und $\sigma=$ $a \cup \pi(\alpha)$ ist eine Ebene. Die Polare einer beliebigen Geraden $b \subseteq \alpha$ geht durch den Punkt $\pi(\alpha)$. Für eine solche Gerade $b$ ist die Aussage $b \sim a$ also gleichwertig damit, daß $\pi(b)$ in der Ebene $\sigma$ liegt. Die Aussage $\pi(b) \subseteq \sigma$ ist aber ihrerseits gleichwertig damit, daß $\pi(\sigma) \in b$, also $b$ durch den Punkt $\pi(a \cup \pi(\alpha))$ geht.

Von einer beliebigen Ecke $P_{i}$ eines Tetraeders $\Delta=P_{0} P_{1} P_{2} P_{3}$ aus existiert ein eindeutig bestimmtes Lot auf die gegenüberliegende Seitenfläche $P_{j} P_{k} P_{l}$, vorausgesetzt daß $P_{i} \neq$ $\pi\left(P_{j} P_{k} P_{l}\right)$. Wir nennen ein solches Lot eine Höhe im Tetraeder $\Delta$ und bezeichnen die durch $P_{i}$ gehende Höhe wie vorher mit $h_{i}$. Den Pol $\pi\left(P_{j} P_{k} P_{l}\right)$ der Seitenfläche $P_{j} P_{k} P_{l}$ bezeichnen wir mit $Q_{i}$.

7.5 Hilfssatz. Sei $\{i, j, k, l\}=\{0,1,2,3\}$ und im Tetraeder $P_{0} P_{1} P_{2} P_{3}$ gelte $P_{i} \neq Q_{i}$ und $P_{j} \neq Q_{j}$. Die Höhen $h_{i}$ und $h_{j}$ gehen genau dann durch einen gemeinsamen Punkt, wenn $P_{i} P_{j} \sim P_{k} P_{l}$

Beweis. Die Geraden $P_{i} Q_{i}=h_{i}$ und $P_{j} Q_{j}=h_{j}$ haben genau dann einen Punkt gemeinsam, wenn $P_{i} P_{j}$ und $Q_{i} Q_{j}$ einen Punkt gemeinsam haben. Die Gerade $Q_{i} Q_{j}$ ist aber die Polare von $P_{k} P_{l}$.

Wir nennen ein Tetraeder zulässig, wenn keine seiner Kanten zur gegenüberliegenden konjugiert ist. Daraus folgt insbesondere, daß keine Ecke mit dem Pol der gegenüberliegenden Seitenfläche zusammenfällt.

7.6 Satz von Steiner. In jedem zulässigen Tetraeder liegen die Höhen in einer von den beiden Geradenscharen eines Hyperboloids.

Dieser Satz findet sich in [12, S. 153] noch ohne die einschränkende Voraussetzung, daß das Tetraeder zulässig sein muß; Ex. 7 in [4, Vol III, S. 41] enthält die korrekte Formulierung. Natürlich ist die Gültigkeit des Satzes in einem beliebigen projektiven Raum $\mathcal{P}$ mit Polarität nicht zu erwarten. Uns geht es hier um die Bedingungen für seine Gültigkeit.

7.7 Steinersche Bedingung. Durch jede Ecke eines beliebigen zulässigen Tetraeders geht eine Treffgerade der vier Höhen. 
Für Dreikante übernehmen wir unverändert die im vorigen Abschnitt eingeführten Bezeichnungsweisen. Neben der Steinerschen Bedingung interessieren uns noch die beiden folgenden:

7.8 Projektive Dreikantbedingung. In jedem zulässigen Dreikant haben die zu den gegenüberliegenden Seitenflächen orthogonalen Ebenen durch die Kanten eine Gerade gemeinsam.

7.9 Projektive Tetraederbedingung. Sind in einem Tetraeder zwei Paare gegenüberliegender Kanten zueinander konjugiert, so gilt dies auch für das dritte Paar.

Abgesehen von dem Sonderfall des projektiven Raumes über dem Körper mit zwei Elementen, ergibt sich die Existenz von zulässigen Tetraedern ähnlich wie im Hilfssatz 6.1 des vorigen Kapitels.

7.10 Hilfssatz. Sei $P_{0}$; abc ein zulässiges Dreikant. Wenn es auf jeder Geraden mindestens vier Punkte gibt, so lassen sich Punkte $P_{1} \in a, P_{2} \in b$ und $P_{3} \in c$ finden, die zusammen mit $P_{0}$ ein zulässiges Tetraeder bilden.

Beweis. Seien $\alpha=b \cup c, \beta=c \cup a$ und $\gamma=a \cup b$ die Seitenflächen des Dreikants. Wir betrachten wie in Hilfssatz 7.4 die Punkte $S_{1}=\pi(a \cup \pi(\alpha)), S_{2}=\pi(b \cup \pi(\beta))$ und $S_{3}=\pi(c \cup \pi(\gamma))$. Die zu $a$ konjugierten Geraden der Ebene $\alpha$ gehen alle durch den Punkt $S_{1}$, und entsprechendes gilt für $\beta$ und $S_{2}$ sowie $\gamma$ und $S_{3}$. Auf der Geraden $a$ gibt es nun jedenfalls einen Punkt $P_{1}$, der von $P_{0}, S_{2}$ und $S_{3}$ verschieden ist. Durch $P_{1}$ geht in der Ebene $\beta$ genau eine Gerade, die zu $b$ konjugiert ist, nämlich $P_{1} S_{2}$. Somit gibt es in der Ebene $\beta$ noch mindestens zwei von $a$ verschiedene Geraden durch $P_{1}$, die zu $b$ nicht konjugiert sind. Ihre Schnittpunkte mit $c$ seien $P_{3}$ und $P_{3}^{\prime}$. Ebenso gibt es in $\gamma$ noch zwei von $a$ verschiedene Geraden durch $P_{1}$, die zu $c$ nicht konjugiert sind. Ihre Schnittpunkte mit $b$ bezeichnen wir mit $P_{2}$ und $P_{2}^{\prime}$. Unter den vier Geraden $P_{2} P_{3}, P_{2} P_{3}^{\prime}, P_{2}^{\prime} P_{3}, P_{2}^{\prime} P_{3}^{\prime}$, die keinen Punkt gemeinsam haben, geht mindestens eine nicht durch den Punkt $S_{1}$. Notfalls nach Umbenennung der Punkte können wir annehmen, dies sei die Gerade $P_{2} P_{3}$. Dann ist also $P_{2} P_{3}$ nicht konjugiert zur Geraden $a$, und $P_{0} P_{1} P_{2} P_{3}$ ist ein zulässiges Tetraeder.

Satz 2 Sei $\mathcal{P}$ ein dreidimensionaler projektiver Raum mit Polarität $\pi, K$ der zugehörige Koordinatenkörper und $f$ eine in dem zugrundeliegenden Vektorraum über $K$ definierte Semibilinearform, welche $\pi$ induziert. Dann sind folgende Aussagen äquivalent:

i) In $\mathcal{P}$ ist die Steinersche Bedingung erfüllt.

ii) In $\mathcal{P}$ ist die projektive Dreikantbedingung erfüllt.

iii) In $\mathcal{P}$ gilt der projektive Satz vom Höhenschnittpunkt in jedem Punktbüschel, dessen Träger nicht auf seiner eigenen Polaren liegt. Ist $\pi$ ein Nullsystem, so gilt darüber hinaus $\operatorname{char}(K)=2$.

iv) In $\mathcal{P}$ gilt der projektive Satz vom Höhenschnittpunkt in jeder Ebene, die ihren Pol nicht enthält. Ist $\pi$ ein Nullsystem, so gilt darüber hinaus $\operatorname{char}(K)=2$. 
v) $K$ ist kommutativ, und $f$ ist eine symmetrische Bilinearform.

vi) In $\mathcal{P}$ ist die zur projektiven Dreikantbedingung duale Bedingung erfüllt.

vii) In $\mathcal{P}$ ist die projektive Tetraederbedingung erfüllt.

viii) In $\mathcal{P}$ gilt der Satz von Steiner.

Zum Beweis des Satzes benötigen wir noch einige Hilfssätze.

7.11 Hilfssatz. In dem dreidimensionalen projektiven Raum über dem Körper mit zwei Elementen ist die Dreikantbedingung für jede Polarität $\pi$ erfüllt.

Beweis. Dies folgt aus dem Hilfssatz in $\$ 5$ angewandt auf eine beliebige Büschelebene $\mathcal{E}(P)$.

7.12 Hilfssatz. Ist $\pi$ ein Nullsystem, so folgt aus der Dreikantbedingung die Aussage $\operatorname{char}(K)=2$.

Beweis. Wir betrachten einen Punkt $P$ und interpretieren die Ebenen durch den Punkt $P$ als Geraden der Büschelebene $\mathcal{E}(P)$. Die Ebene $\pi(P)$ geht durch $P$ und wir betrachten $\mathcal{E}(P)$ als affine Ebene mit der unendlich fernen Geraden $\pi(P)$. Das Senkrechtstehen der durch $P$ gehenden Ebenen interpretieren wir als Senkrechtstehen der Geraden in $\mathcal{E}(P)$. Da $\pi$ ein Nullsystem ist, so liegt der Pol einer beliebigen durch $P$ gehenden und von $\pi(P)$ verschiedenen Ebene $\alpha$ in $\pi(P) \cap \alpha$ und ist von $P$ verschieden. Zwei Ebenen durch den Punkt $P$ stehen daher senkrecht aufeinander, wenn ihre Schnittgerade in $\pi(P)$ enthalten ist. Dies bedeutet nichts anderes, als daß sich in $\mathcal{E}(P)$ das Senkrechtstehen von Geraden auf die Parallelität reduziert. Damit folgt die Behauptung aus der Bemerkung in $\S 4$.

7.13 Hilfssatz. Ist $\pi$ ein Nullsystem, so folgt aus der Tetraederbedingung die Aussage $\operatorname{char}(K)=2$.

Beweis. Sei $\delta$ eine beliebige Ebene und sei $S=\pi(\delta)$. Es gilt $S \in \delta$, weil $\pi$ ein Nullsystem ist. Sind $a, b, c$ drei nicht durch $S$ gehende Geraden von $\delta$, die keinen Punkt gemeinsam haben, so gehen die drei Geraden $\pi(a), \pi(b), \pi(c)$ durch $S$, sind nicht in $\delta$ enthalten und haben keine Ebene gemeinsam. Wir setzen $A=b \cap c, B=c \cap a$ und $C=a \cap b$. Sei $\gamma$ eine von $\delta$ verschiedene Ebene durch die Gerade $c$. Sie schneidet $\pi(a)$ und $\pi(b)$ in Punkten $X$ bzw. $Y$, und wir setzen $D=A X \cap B Y$. Im Tetraeder $A B C D$ gilt dann $A D \sim B C$ und $B D \sim A C$. Aus der Tetraederbedingung folgt $C D \sim A B$. Weiter folgt noch, daß jede der drei Ebenen $A \cup \pi(a), B \cup \pi(b)$ und $C \cup \pi(c)$ die Gerade $D S$ enthält.

Betrachten wir nun die Ebenen $\pi(A)=\pi(b) \cup \pi(c), \pi(B)=\pi(c) \cup \pi(a)$ und $\pi(C)=$ $\pi(a) \cup \pi(b)$. Da $\pi$ ein Nullsystem ist, gilt z.B. $A \in \pi(A)$. Daraus folgt $A \cup \pi(a) \perp$ $\pi(A)$. Ebenso folgt $B \cup \pi(b) \perp \pi(B)$ und $C \cup \pi(c) \perp \pi(C)$. Stehen aber zwei durch $S$ gehende und von $\delta$ verschiedene Ebenen aufeinander senkrecht, so schneiden sie sich in einer Geraden, die in $\delta$ enthalten ist. Betrachten wir nun die Büschelebene $\mathcal{E}(S)$ als affine Ebene mit Ferngerade $\delta$, so ist das Senkrechtstehen von Geraden in $\mathcal{E}(S)$ nichts anderes als 
Parallelität. Die Geraden $\pi(a), \pi(b), \pi(c)$ bilden aber in $\mathcal{E}(S)$ ein Dreieck, dessen Höhen durch den durch $D S$ repräsentierten Punkt gehen. Daraus folgt $\operatorname{char}(K)=2$.

Beweis von Satz 2. i) $\rightarrow$ ii). Es sei $P_{0}$; $a b c$ ein zulässiges Dreikant mit den Seitenflächen $\alpha=b \cup c, \beta=c \cup a$ und $\gamma=a \cup b$. Wir bezeichnen mit $\alpha_{1}, \beta_{1}, \gamma_{1}$ die auf $\alpha, \beta$ bzw. $\gamma$ senkrecht stehenden Ebenen, die durch $a, b$ bzw. $c$ gehen.

Wenn es Punkte $P_{1} \in a, P_{2} \in b, P_{3} \in c$ gibt, die zusammen mit dem Punkt $P_{0}$ ein zulässiges Tetraeder bilden, so sei $t$ die durch $P_{0}$ gehende Treffgerade der vier Höhen dieses Tetraeders. Wir wollen zeigen, daß $t \subseteq \alpha_{1}$. Es ist $\alpha_{1}=a \cup \pi(\alpha)=P_{0} P_{1} \pi(\alpha)=$ $a \cup h_{1}$. Da $t$ durch den Punkt $P_{0}$ geht und die Höhe $h_{1}$ trifft, folgt $t \subseteq \alpha_{1}$. Auf ähnliche Weise zeigt man, daß auch $t \subseteq \beta_{1}$ und $t \subseteq \gamma_{1}$ gilt, und somit haben die drei Ebenen $\alpha_{1}$, $\beta_{1}$ und $\gamma_{1}$ die Gerade $t$ gemeinsam.

Nun haben wir also nur noch zu zeigen, daß solche Punkte $P_{1}, P_{2}, P_{3}$ existieren. Wegen Hilfssatz 7.11 können wir dabei annehmen, daß es auf jeder Geraden mindestens vier Punkte gibt. Dann folgt die Existenz der Punkte $P_{1}, P_{2}, P_{3}$ aus Hilfssatz 7.10.

ii) $\rightarrow$ iii). Sei $P$ ein Punkt, der nicht auf seiner Polaren $\pi(P)$ liegt. Die Geraden der zum Büschel $(P)$ gehörigen projektiven Ebene $\mathcal{E}(P)$ sind also die Ebenen $\alpha$ durch den Punkt $P$, die Punkte von $\mathcal{E}(P)$ sind die Geraden $a$ durch $P$. Der Punkt $a$ und die Gerade $\alpha$ sind Pol und Polare in $\mathcal{E}(P)$, wenn $\pi(\alpha) \in a$, d.h. wenn $a \perp \alpha$. Ein Dreieck der Ebene $\mathcal{E}(P)$ ist also gegeben durch ein Dreikant mit Spitze im Punkt $P$, und die Bedingung, daß keine Ecke des Dreiecks mit dem Pol der Gegenseite zusammenfällt, entspricht der Aussage, daß in dem Dreikant keine Kante auf der gegenüberliegenden Seitenfläche senkrecht steht. Aus der Dreikantbedingung folgt daher der projektive Satz vom Höhenschnittpunkt, d.h. der erste Teil der Aussage iii).

Der zweite Teil der Aussage iii) folgt aus Hilfssatz 7.12.

iii) $\rightarrow$ iv). Dies folgt aus Dualitätsgründen.

iv) $\rightarrow$ v). Wir betrachten zuerst den Fall, daß $\pi$ kein Nullsystem ist. Sei also $\alpha$ eine Ebene, die ihren Pol $P=\pi(\alpha)$ nicht enthält. Nach [11, Satz 19, S. 65] folgt aus dem projektiven Satz vom Höhenschnittpunkt in der Ebene $\alpha$, daß die durch $\pi$ induzierte Polarität $\pi_{\alpha}$ projektiv ist. Sei $g$ eine beliebige Gerade von $\alpha$. Dann geht $\pi(g)$ durch den Punkt $P$. Die Abbildung $X \rightarrow \pi_{\alpha}(X)=X \cap \alpha$ für $X \in g$ ist eine Projektivität der Punktreihe $g$ auf das Geradenbüschel mit Träger $\pi(g) \cap \alpha$. Somit ist die Abbildung $X \rightarrow \pi(X)$ eine projektive Abbildung der Punktreihe $g$ auf das Ebenenbüschel mit Träger $\pi(g)$. Daraus folgt, daß die Polarität $\pi$ projektiv ist. Daher ist $K$ kommutativ, und weil $\pi$ kein Nullsystem ist, ist die Form $f$ symmetrisch.

Ist nun $\pi$ ein Nullsystem, so ist $K$ kommutativ und $f$ schiefsymmetrisch. Da in diesem Fall aber $\operatorname{char}(K)=2$ gilt, so ist $f$ zugleich auch symmetrisch.

v) $\rightarrow$ vi). Die zur Dreikantbedingung duale Bedingung lautet:

(A) Sei $A_{1} B_{1} C_{1}$ ein Dreieck in einer Ebene $\alpha$ und sei keine Dreiecksseite in der Polaren der gegenüberliegenden Ecke enthalten. Dann liegen die Punkte $P=\pi\left(A_{1}\right) \cap B_{1} C_{1}$, $Q=\pi\left(B_{1}\right) \cap C_{1} A_{1}$ und $R=\pi\left(C_{1}\right) \cap A_{1} B_{1}$ kollinear. 
Seien $u, v, w$ unabhängige Vektoren, so daß $A_{1}=K u, B_{1}=K v$ und $C_{1}=K w$. Sei ferner $P=K p, Q=K q, R=K r$. Nach Voraussetzung gilt

$$
f(p, u)=f(q, v)=f(r, w)=0 .
$$

Wir setzen $f(u, v)=f(v, u)=\alpha, f(u, w)=f(w, u)=\beta$ und $f(v, w)=f(w, v)=$ $\gamma$. Von den Körperelementen $\alpha, \beta, \gamma$ kann jeweils höchstens eines verschwinden, da sonst eine der Voraussetzungen $B_{1} C_{1} \nsubseteq \llbracket \pi\left(A_{1}\right)$ usw. verletzt würde. Daher sind $p_{1}=\beta v-\alpha w$, $q_{1}=\gamma u-\alpha w, r_{1}=\gamma u-\beta v$ von Null verschiedene Vektoren. Die Punkte $K p_{1}, K q_{1}$, $K r_{1}$ liegen respektive auf $B_{1} C_{1}, C_{1} A_{1}$ und $A_{1} B_{1}$. Da nun, wie man leicht nachprüft, auch die Gleichungen $f\left(p_{1}, u\right)=f\left(q_{1}, v\right)=f\left(r_{1}, w\right)=0$ erfüllt sind, so folgt, daß die Vektoren $p, q, r$ den $p_{1}, q_{1}, r_{1}$ proportional sind. Letztere stehen aber in der Relation $p_{1}-q_{1}+r_{1}=0$. Also sind $P, Q, R$ kollinear.

vi) $\rightarrow$ vii). Es ist zu zeigen: Aus (A) folgt die Tetraederbedingung. Dies geht im Wesentlichen wie in [4, Vol. III, Ex. 5, S. 34]. Wir betrachten ein Tetraeder $A B C D$ und das dazu polare Tetraeder mit den Ecken $A_{1}=\pi(B C D), B_{1}=\pi(C D A), C_{1}=\pi(D A B)$ und $D_{1}=\pi(A B C)$. Wir nehmen $A C \sim B D$ und $A B \sim C D$ an und haben zu zeigen $A D \sim B C$. Diese Annahme bedeutet, daß die Durchschnitte $A C \cap A_{1} C_{1}$ und $A B \cap A_{1} B_{1}$ nicht leer sind und wir müssen zeigen, daß auch $B C \cap B_{1} C_{1}$ nicht leer ist. Wir dürfen annehmen, da $\beta B_{1} C_{1}$ nicht in der Ebene $B C D$ enthalten ist. Ferner kann angenommen werden, daß $A_{1} \neq A, B_{1} \neq B$ und $C_{1} \neq C$. Denn in allen drei Fällen ist $B C \sim A D$. Aus $A_{1} B_{1} \subseteq D A B=\pi\left(C_{1}\right)$ folgt $C_{1} \in \pi\left(A_{1} B_{1}\right)=C D$. Da $C_{1} A_{1}$ die Gerade $C A$ schneidet, gilt dann auch $C_{1} A_{1} \subseteq C D A$. Es folgt $B_{1} \in \pi\left(C_{1} A_{1}\right)=B D$. Daher schneiden sich $B C$ und $B_{1} C_{1}$, und wir sind fertig. Auf ähnliche Weise kommen wir zum Ziel, wenn $A_{1} C_{1} \subseteq D A C$. Also dürfen wir nun annehmen, daß die Geraden $A_{1} B_{1}, B_{1} C_{1}, C_{1} A_{1}$ nicht in den Ebenen $A B D, B C D$ bzw. $A D C$ enthalten sind. Es existieren also die Schnittpunkte $R=A B D \cap A_{1} B_{1}, Q=A D C \cap A_{1} C_{1}$ und $P=B C D \cap B_{1} C_{1}$. Nach Aussage (A) liegen die drei Punkte $P, Q, R$ in einer Geraden $g$. Aus der Voraussetzung, daß die Durchschnitte $A C \cap A_{1} C_{1}$ und $A B \cap A_{1} B_{1}$ nicht leer sind, folgt $A C \cap A_{1} C_{1}=Q$ und $A B \cap A_{1} B_{1}=R$. Die beiden Punkte $Q$ und $R$ liegen mithin in der Ebene $A B C$. Wären sie gleich, so müßten sie beide mit dem Punkt $A$ zusammenfallen, und es würde auch $A_{1}=A$ folgen, was wir aber bereits ausgeschlossen hatten. Also liegen die Gerade $Q R$ und folglich auch der Punkt $P$ in der Ebene $A B C$. Da $P$ auch in der Ebene $B C D$ liegt, schneiden sich die Geraden $B C$ und $B_{1} C_{1}$ im Punkt $P$ und die Tetraederbedingung ist bewiesen.

vii) $\rightarrow$ iv). Sei $\alpha$ eine Ebene, die ihren Pol $\pi(\alpha)$ nicht enthält, und sei $A B C$ ein in $\alpha$ enthaltenes Dreieck, bei dem keine Ecke mit dem Pol ihrer Gegenseite bezüglich der induzierten Polarität $\pi_{\alpha}$ zusammenfällt. Der Pol der Seite $a=B C$ bezüglich $\pi_{\alpha}$ ist definiert als $\pi_{\alpha}(a)=\pi(a) \cap \alpha$. Wir bezeichnen ihn mit $A_{1}$. Ferner setzen wir $b=A C$ und $B_{1}=\pi_{\alpha}(b)=\pi(b) \cap \alpha$ sowie $c=A B$ und $C_{1}=\pi_{\alpha}(c)=\pi(c) \cap \alpha$. Sei $H$ der Schnittpunkt der Höhen $A A_{1}$ und $B B_{1}$. Wir wählen auf der Geraden $H \pi(\alpha)$ einen von $H$ und $\pi(\alpha)$ verschiedenen Punkt $D$. In dem Tetraeder $A B C D$ gilt $A D \sim B C=a$, weil $A D$ ebenso wie $\pi(a)=A_{1} \pi(\alpha)$ in der Ebene $A D \pi(\alpha)$ liegen. Ebenso gilt $B D \sim A C=b$, weil $B D$ und $\pi(b)=B_{1} \pi(\alpha)$ beide in der Ebene $B D \pi(\alpha)$ liegen. Aus der Tetraederbedingung folgt, daß auch $C D \sim A B=c$. Dies bedeutet, daß auch $C D$ und $\pi(c)=C_{1} \pi(\alpha)$ 
in einer Ebene liegen. Folglich geht auch die dritte Höhe $C C_{1}$ durch den Punkt $H$. Damit ist iv) bewiesen, wenn $\pi$ kein Nullsystem ist.

Ist nun $\pi$ ein Nullsystem, so folgt $\operatorname{char}(K)=2$ nach Hilfssatz 7.13.

iv) $\rightarrow$ ii). Die Äquivalenz der Aussagen iv)-vii) ist nun bewiesen. Die Aussagen vi) und ii) sind dual zueinander und somit ebenfalls äquivalent.

ii) $\rightarrow$ i). Sei $P_{0} P_{1} P_{2} P_{3}$ ein zulässiges Tetraeder. Wir betrachten das Dreikant mit den Kanten $a=P_{0} P_{1}, b=P_{0} P_{2}$ und $c=P_{0} P_{3}$. Es ist zulässig. Sei also $t$ die Gerade, die den durch die Kanten gehenden und zu den gegenüberliegenden Seitenflächen orthogonalen Ebenen gemeinsam ist. Sei beispielsweise $\alpha$ die durch $a$ und den Pol $Q_{1}$ der Seitenfläche $b \cup c=P_{0} P_{2} P_{3}$ gehende Ebene. Sie enthält offensichtlich die Höhe $h_{1}=P_{1} Q_{1}$. Somit sind $h_{1}$ und $t$ Treffgeraden. Auf ähnliche Weise folgt, daß auch $h_{2}$ und $t$ sowie $h_{3}$ und $t$ Treffgeraden sind. Da auch $h_{0}$ und $t$ Treffgeraden sind, ist $t$ eine Treffgerade aller vier Höhen. Aus Symmetriegründen folgt, daß auch durch die übrigen Ecken Treffgeraden der vier Höhen existieren. Damit ist i) bewiesen.

i) $\rightarrow$ viii). Wie wir bereits wissen, hat i) zur Folge, daß der Körper $K$ kommutativ und daß $f$ eine symmetrische Bilinearform ist. Um zu zeigen, daß die Höhen in einem Hyperboloid liegen, würde es also genügen, die Existenz von mindestens drei Treffgeraden nachzuweisen. Die durch die Ecken des Tetraeders gehenden Treffgeraden sind nur in dem einen Sonderfall weniger als drei, wenn je zwei von ihnen mit einer Kante zusammenfallen. Die beiden Kanten liegen dann natürlich einander gegenüber.

In diesem Sonderfall sei $H_{i}$ der Fußpunkt der Höhe $h_{i}, i=0, \ldots, 3$. Wir können also zum Beispiel annehmen, daß $H_{2}, H_{3}$ auf der Kante $P_{0} P_{1}$ und $H_{0}, H_{1}$ auf der Kante $P_{2} P_{3}$ liegen. Wir betrachten nun das zu $P_{0}, P_{1}, P_{2}, P_{3}$ polare Tetraeder mit den Ecken $Q_{0}=$ $\pi\left(P_{1} P_{2} P_{3}\right), Q_{1}=\pi\left(P_{0} P_{2} P_{3}\right)$ usw. Die Höhen des polaren Tetraeders sind identisch mit den Höhen des ursprünglichen Tetraeders. Deshalb folgt aus der Steinerschen Bedingung, daß auch durch jede Ecke des polaren Tetraeders eine Treffgerade der vier Höhen geht. Ist nun eine der Ecken des polaren Tetraeders von dem entsprechenden Höhenfußpunkt verschieden, z.B. $Q_{0} \neq H_{0}$, so geht durch diese Ecke eine weitere Treffgerade, die mit keiner Kante zusammenfallen kann.

Es bleibt also nur noch der spezielle Fall zu betrachten, daß $H_{i}=Q_{i}$ für $i=0, \ldots, 3$. Es genügt nun der Nachweis, daß die Punktequadrupel $P_{0}, P_{1}, Q_{2}, Q_{3}$ und $Q_{0}, Q_{1}, P_{2}, P_{3}$ projektiv aufeinander bezogen sind (s. Schröter [12, S. 87]). Wir betrachten für die Punktreihe $P_{0} P_{1}$ nacheinander zwei Operationen: zuerst ersetzen wir einen beliebigen Punkt $X$ durch seine Polare $\pi(X)$, dann die Ebene $\pi(X)$ durch ihren Schnittpunkt mit der Geraden $P_{2} P_{3}$. Da $\pi$ durch eine symmetrische Bilinearform dargestellt wird, ist die betrachtete Abbildung $X \rightarrow \pi(X) \cap P_{2} P_{3}$ eine Projektivität. Man prüft leicht nach, daß sie das Punktequadrupel $P_{0}, P_{1}, Q_{2}, Q_{3}$ in das Quadrupel $Q_{1}, Q_{0}, P_{3}, P_{2}$ überführt. Damit sind auch die Quadrupel $P_{0}, P_{1}, Q_{2}, Q_{3}$ und $Q_{0}, Q_{1}, P_{2}, P_{3}$ projektiv aufeinander bezogen. Somit sind die Höhen $h_{0}=P_{0} Q_{0}, h_{1}=P_{1} Q_{1}, h_{2}=P_{2} Q_{2}$ und $h_{3}=P_{3} Q_{3}$ als Verbindungsgeraden aufeinander bezogener Punkte Erzeugende eines Hyperboloids.

vii) $\rightarrow$ i). Dies ergibt sich aus der Definition eines Hyperboloids.

Damit ist Satz 2 bewiesen. 


\section{Bemerkungen.}

1. Im affinen oder projektiven Raum über dem Körper mit zwei Elementen gibt es keine zulässigen Tetraeder. Denn nach Satz 1 oder nach Satz 2 gilt der Satz von Steiner. Wäre nun $\Delta$ ein zulässiges Tetraeder, so lägen die vier Höhen als Erzeugende in einem Hyperboloid. Dies ist aber unmöglich, denn weil die Geraden nur drei Punkte enthalten, so bestehen auch die Erzeugendenscharen aus jeweils nur drei Geraden.

2. Der Satz von Steiner bleibt auch in der absoluten Geometrie im Sinne von Ahrens [1] gültig. Zum Beweis kann man den absoluten Raum auf kanonische Weise wie von Ahrens beschrieben in einen projektiven Raum einbetten und dann im verallgemeinerten euklidischen Fall Satz 1, im verallgemeinerten hyperbolischen oder elliptischen Fall Satz 2 anwenden.

\section{Literatur}

[1] Ahrens, J.: Begründung der absoluten Geometrie des Raumes aus dem Spiegelungsbegriff. Math. Z. 71 (1959), 154-185.

[2] Baer, R.: Linear Algebra and Projective Geometry. Academic Press, New York 1952.

[3] Baer, R.: The fundamental theorems of elementary geometry. Trans. Amer. Math. Soc. 56 (1944), 94-129.

[4] Baker, H.F.: Principles of Geometry. Vol. I-VI. Cambridge University Press, 1922.

[5] Berger, M.: Geometry. (Translated from the French by Cole, M.; Levy, S.). Springer-Verlag, Berlin, Heidelberg etc. 1987.

[6] Dickson, L.E.: Linear Groups. Teubner, 1903.

[7] Hermes, O.: Journal für Math. 56 (1858), 241.

[8] Herzer, A.: Eine Verallgemeinerung des Satzes von Dandelin. Elem. Math. 27 (1972), 52-56.

[9] Joachimsthal, F.J.: Zur Theorie der dreiseitigen Pyramide. Grunert Arch. Bd. 32 (1859), 107-111.

[10] Lenz, H.: Über die Einführung einer absoluten Polarität in die projektive und affine Geometrie des Raumes. Math. Ann. 128 (1954), 363-372.

[11] Lenz, H.: Zur Begründung der analytischen Geometrie. Bayer. Akad. Wiss. Math.-Natur. Kl. (1954), 17-72.

[12] Schröter, H.: Theorie der Oberflächen zweiter Ordnung und der Raumkurven dritter Ordnung als Erzeugnisse projektivischer Gebilde. B.G. Teubner Verlag, Leipzig 1880.

[13] Steiner, J.: Journal für Math. 2 (1827), 97.

[14] Steiner, J.: Systematische Entwickelung der Abhängigkeit geometrischer Gestalten von einander. Berlin 1832.

Adolf Schleiermacher

Rablstr. 18

D-81669 München, Deutschland

e-mail: adsle@aol.com

${ }^{1}$ Zusatz bei der Korrektur: Zum Höhenhyperboloid im euklidischen Raum siehe auch H. Havlicek und G. Weiss in Amer. Math. Monthly 110, No. 8 (2003), 679-693. Der Autor dankt Herrn V. Pambuccian für diesen Hinweis. 